ANNALES

POLONICI MATHEMATICI

$87(2005)$

\title{
Non-uniruledness and the cancellation problem
}

\author{
by ROBERT DryŁo (Kraków)
}

To the memory of Professor Stanistaw Lojasiewicz

\begin{abstract}
Using the notion of uniruledness we indicate a class of algebraic varieties which have a stronger version of the cancellation property. Moreover, we give an affirmative solution to the stable equivalence problem for non-uniruled hypersurfaces.
\end{abstract}

Introduction. An algebraic variety $X$ has the strong cancellation property if every isomorphism $f: Y \times \mathbb{C}^{m} \rightarrow X \times \mathbb{C}^{m}$, where $Y$ is an algebraic variety, satisfies the condition: for each $y \in Y$ there exists $x \in X$ such that $f\left(\{y\} \times \mathbb{C}^{m}\right)=\{x\} \times \mathbb{C}^{m}$ (then clearly $f$ induces an isomorphism between $X$ and $Y$ ). Iitaka and Fujita proved in [3] that an algebraic variety of non-negative logarithmic Kodaira dimension has the strong cancellation property.

Using mainly the notion of uniruledness we will give some other conditions guaranteeing this property. Namely, we will show that an affine variety none of whose components is $\mathbb{C}$-uniruled has the strong cancellation property (Theorem 1); in particular, affine irreducible varieties with two non-uniruled components at infinity (Corollary 1) and affine unirational varieties with a non-uniruled component at infinity have this property (Theorem 2).

We will also deal with the stable equivalence problem.

As in [7], affine varieties $X, Y$ in $\mathbb{C}^{n}$ are said to be equivalent if there exists a polynomial automorphism of $\mathbb{C}^{n}$ carrying $X$ onto $Y$. Moreover, $X, Y$ are said to be stably equivalent if for some $m \in \mathbb{N}$ the cylinders $X \times \mathbb{C}^{m}$ and $Y \times \mathbb{C}^{m}$ are equivalent (in $\mathbb{C}^{n+m}$ ).

Makar-Limanov, van Rossum, Shpilrain and Yu in [7] stated and solved for curves in $\mathbb{C}^{2}$ (in fact they proved a general result for polynomials of two variables over an arbitrary field of characteristic zero) the following

Stable equivalence Problem. Are two stably equivalent hypersurfaces in $\mathbb{C}^{n}$ equivalent?

2000 Mathematics Subject Classification: Primary 14R10; Secondary 14E05.

Key words and phrases: uniruled variety, cancellation property, stable equivalence. 
Shpilrain and $\mathrm{Yu}$ in [10] solved this problem when one of the hypersurfaces is the set of zeros of a so-called test polynomial (see $[1,5]$ ).

We give an affirmative answer when one of the hypersurfaces is nonuniruled (Corollary 2).

Now we give some basic definitions.

An irreducible variety $X$ of dimension $n>0$ is said to be uniruled if there exists a variety $W$ of dimension $n-1$ and a dominant rational map $W \times \mathbb{C} \rightarrow X$. A reducible affine variety is said to be uniruled if all its irreducible components are uniruled.

It is well known that a variety is uniruled if and only if through its generic point passes a rational curve.

A generic hypersurface in $\mathbb{C}^{n}$ of degree $d>n$ is an example of a nonuniruled variety (see [4]).

A similar notion is that of $\mathbb{C}$-uniruledness introduced by Jelonek in [6]. An affine irreducible variety $X$ of dimension $n>0$ is said to be $\mathbb{C}$-uniruled if there exists a variety $W$ of dimension $n-1$ and a dominant polynomial map $W \times \mathbb{C} \rightarrow X$. A reducible affine variety is said to be $\mathbb{C}$-uniruled if all its irreducible components are $\mathbb{C}$-uniruled.

The $\mathbb{C}$-uniruledness means exactly that through a generic point of the variety passes an affine parametric line (see [6]).

By a hypersurface in an irreducible algebraic variety we mean an algebraic subvariety of pure codimension one.

Main results. We begin by introducing the notion of verticality. Let $X, Y$ be algebraic varieties. A regular mapping $f: Y \times \mathbb{C}^{m} \rightarrow X \times \mathbb{C}^{m}$ is called vertical over $a \in Y$ if there exists $b \in X$ such that $f\left(\{a\} \times \mathbb{C}^{m}\right) \subset\{b\} \times \mathbb{C}^{m}$. Moreover, we say that $f$ is vertical over a subset $Z$ of $Y$ if it is vertical over each point of $Z$.

We define $Z_{f}=\{y \in Y \mid f$ is vertical over $y\}$. It is a Zariski-closed subset of $Y$. Indeed, we can suppose that $X, Y \subset \mathbb{C}^{n}$. If $f=\left(f_{1}, \ldots, f_{n+m}\right)$ then

$$
\begin{aligned}
Z_{f} & =\left\{y \in Y \mid f_{1}(y, \cdot), \ldots, f_{n}(y, \cdot) \text { are constant on } \mathbb{C}^{m}\right\} \\
& =\bigcap_{i=1}^{n} \bigcap_{z, t \in \mathbb{C}^{m}}\left\{y \in Y \mid f_{i}(y, z)-f_{i}(y, t)=0\right\} .
\end{aligned}
$$

Obviously the strong cancellation property for a variety $X$ is equivalent to saying that $Z_{f}=Y$ for every variety $Y$ and every isomorphism $f: Y \times \mathbb{C}^{m} \rightarrow$ $X \times \mathbb{C}^{m}$. Now we are ready to prove

THEOREM 1. An affine variety none of whose components is $\mathbb{C}$-uniruled has the strong cancellation property.

Proof. Let a variety $X$ satisfy the assumptions. We can take $X$ to be irreducible. Suppose that $Z_{f} \neq Y$ for some variety $Y$ and some isomorphism 
$f: Y \times \mathbb{C}^{m} \rightarrow X \times \mathbb{C}^{m}$. Put $V=\pi_{X}\left(f\left(Z_{f} \times \mathbb{C}^{m}\right)\right)$, where $\pi_{X}: X \times \mathbb{C}^{m} \rightarrow X$ is the projection. For each $y \in Z_{f}$ we have $f\left(\{y\} \times \mathbb{C}^{m}\right)=\left\{\pi_{X}(f(y))\right\} \times \mathbb{C}^{m}$. This means that $V \times \mathbb{C}^{m}=f\left(Z_{f} \times \mathbb{C}^{m}\right)$ is closed, hence $V$ is closed. Moreover $V \neq X$ because $\operatorname{dim} V=\operatorname{dim} f\left(Z_{f} \times \mathbb{C}^{m}\right)-m=\operatorname{dim} Z_{f}<\operatorname{dim} Y=$ $\operatorname{dim} X$. Hence $X \backslash V=\bigcup_{y \in Y \backslash Z_{f}} \pi_{X}\left(f\left(\{y\} \times \mathbb{C}^{m}\right)\right)$ is a union of affine parametric lines, which is impossible.

Before stating the next results let us explain that the phrase an affine variety $X$ has $k$ non-uniruled components at infinity means that there exists a compactification $\widehat{X}$ of $X$ such that the variety $\widehat{X} \backslash X$ contains at least $k$ non-uniruled components. (It is well known that for an affine variety $X$ and its compactification $\widehat{X}$ the set $\widehat{X} \backslash X$ is a hypersurface in $\widehat{X}$.) It can be shown (see [6] or Lemma 1 below) that an irreducible $\mathbb{C}$-uniruled variety has at most one non-uniruled component at infinity. Hence we have

COROLlary 1. An irreducible affine variety with two non-uniruled components at infinity has the strong cancellation property.

An irreducible affine variety with one non-uniruled component at infinity may not have the strong cancellation property, since we have an isomorphism $(W \times \mathbb{C}) \times \mathbb{C} \ni((x, y), z) \mapsto((x, z), y) \in(W \times \mathbb{C}) \times \mathbb{C}$. However, if we add the unirationality assumption, we have

Theorem 2. Let $X$ be an affine variety of dimension $n>1$. Suppose that $X$ is unirational and has a non-uniruled component at infinity. Then $X$ has the strong cancellation property.

REMARK. It is well known that affine curves have the cancellation property. Furthermore, every affine curve not isomorphic to $\mathbb{C}$ has the strong cancellation property.

Recall that an $n$-dimensional algebraic variety $X$ is unirational if there exists a rational dominant mapping $f: \mathbb{P}^{n} \rightarrow X$.

We will use the following corollary from Hironaka's Theorem [2] (see also [4]):

Lemma 1. Let $X$ be a smooth irreducible algebraic variety with a smooth compactification $\widehat{X}$ such that $\widehat{X} \backslash X$ is a hypersurface in $\widehat{X}$. Let $Y$ be an affine variety with a compactification $\widehat{Y}$ and let $f: X \rightarrow Y$ be a regular, dominant and generically-finite mapping. Then the number of non-uniruled components of the variety $\widehat{Y} \backslash Y$ is not greater than the number of nonuniruled components of the variety $\widehat{X} \backslash X$.

In particular, if affine varieties $X, Y$ are smooth and isomorphic then the numbers of non-uniruled components of the varieties $\widehat{X} \backslash X$ and $\widehat{Y} \backslash Y$ 
are equal, where $\widehat{X}, \widehat{Y}$ are smooth compactifications of $X$ and $Y$ respectively.

Proof. The idea of this proof is due to Z. Jelonek [4]. Applying Hironaka's Theorem to the rational mapping $f: \widehat{X} \rightarrow \widehat{Y}$ we obtain a mapping $\sigma$ : $B \rightarrow \widehat{X}$, which is a composition of a sequence of blowing-ups along smooth centers such that the mapping $f \circ \sigma: B \rightarrow \widehat{Y}$ is regular on $B$. An elementary property of a blowing-up is that the exceptional divisor $E$ of $\sigma$ (i.e. $E$ is a hypersurface in $B$ such that $\operatorname{dim} \sigma(E)<\operatorname{dim} E$ and $\sigma: B \backslash E \rightarrow \widehat{X} \backslash \sigma(E)$ is an isomorphism) is uniruled. Because $\widehat{Y} \backslash Y \subset \operatorname{cl}(f(\widehat{X} \backslash X)) \cup(f \circ \sigma)(E)$, each non-uniruled component of $\widehat{Y} \backslash Y$ is dominated by a non-uniruled component of $\widehat{X} \backslash X$. This concludes the proof.

Proof of Theorem 2. Let $f: Y \times \mathbb{C}^{m} \rightarrow X \times \mathbb{C}^{m}$ be an isomorphism and $\pi_{X}: X \times \mathbb{C}^{m} \rightarrow X, \pi_{Y}: Y \times \mathbb{C}^{m} \rightarrow Y$ denote the projections. For each unirational variety $H$ properly contained in $Y$ and each affine subspace $L \subset \mathbb{C}^{m}$ such that $\operatorname{dim} H+\operatorname{dim} L=n$, the mapping $\pi_{X} \circ f$ : $H \times L \rightarrow X$ is not dominant. Indeed, if $d=\operatorname{dim} H$ then we have a dominant mapping $f_{1}: \mathbb{P}^{d} \rightarrow H$ which is regular on an open set $D$ in $\mathbb{P}^{d}$. Hence we have the regular mapping $\pi_{X} \circ f \circ\left(f_{1} \times\right.$ id $): D \times L \rightarrow X$ which by Lemma 1 cannot be dominant (since $\left(\mathbb{P}^{d} \times \bar{L}\right) \backslash(D \times L)$ is uniruled, where $\bar{L}$ is the projective closure of $L$ ).

Observe that $X$ dominates $Y$ and hence $Y$ is unirational. Indeed, for $y \in Y$ we can find a polynomial mapping $p: X \rightarrow \mathbb{C}^{m}$ whose graph meets $f\left(\{y\} \times \mathbb{C}^{m}\right)$ at some isolated points (if $X \subset \mathbb{C}^{N}$ then $p$ is, for example, the restriction to $X$ of a suitable affine mapping $\left.\mathbb{C}^{N} \rightarrow \mathbb{C}^{m}\right)$. Therefore the mapping $X \ni x \mapsto \pi_{Y}\left(f^{-1}(x, p(x))\right) \in Y$ has a component of dimension zero in the fiber over $y$ and hence is dominant.

Let $g: \mathbb{P}^{n} \rightarrow Y$ be a dominant rational mapping. It is easy to see that there exists a non-empty Zariski-open subset $U$ of $Y$ such that $g: g^{-1}(U) \rightarrow U$ is a proper mapping and a local biholomorphism. Observe that for each variety $V \subset Y$ and $a \in V \cap U$ there exists a unirational variety $H \subset Y$ such that $\operatorname{dim} H+\operatorname{dim} V=n$ and $H$ intersects $V$ isolatedly at $a$. Indeed, we can find an $(n-\operatorname{dim} V)$-dimensional subspace $H^{\prime}$ of $\mathbb{P}^{n}$ such that $H^{\prime} \cap g^{-1}(a) \neq \emptyset$ and $H^{\prime}$ meets $g^{-1}(V)$ isolatedly at each point from $H^{\prime} \cap g^{-1}(a)$. The variety $H=g\left(H^{\prime}\right)$ fulfils our conditions.

To prove the theorem it is enough to show that $f^{-1}$ is vertical over all points from $\pi_{X}\left(f\left(U \times \mathbb{C}^{m}\right)\right)$. Suppose that there exists $x \in \pi_{X}\left(f\left(U \times \mathbb{C}^{m}\right)\right)$ such that the dimension of $\pi_{Y}(W)$ is positive, where $W=f^{-1}\left(\{x\} \times \mathbb{C}^{m}\right)$. Let $\pi: W \rightarrow \mathbb{C}^{m}$ be the projection. Three cases are possible:

CASE 1: $\pi$ is dominant. It is easy to see that we can choose a line $L$ in $\mathbb{C}^{m}$ such that $\pi^{-1}(L)=L^{\prime} \cup T$, where $L^{\prime}$ is an irreducible curve, $L^{\prime} \cap\left(U \times \mathbb{C}^{m}\right) \neq \emptyset$, 
$T$ is closed, $L^{\prime} \not \subset T$ and $\operatorname{dim} \pi_{Y}\left(L^{\prime}\right)=1$. Let $a \in\left(L^{\prime} \backslash T\right) \cap\left(U \times \mathbb{C}^{m}\right)$ and $H$ be a unirational hypersurface in $Y$ which intersects $\pi_{Y}\left(L^{\prime}\right)$ isolatedly at $\pi_{Y}(a)$. Hence $\{a\}$ is a component of $(H \times L) \cap W$. This means that the mapping $\pi_{X} \circ f: H \times L \rightarrow X$ has in the fiber over $x$ a component of dimension zero so it is dominant; a contradiction.

CASE 2: $S=\pi(W)$ has dimension $s<m$ and a fiber of $\pi$ over a generic point of $S$ has the dimension strictly smaller than $n$. Choose $z \in S$ such that $\operatorname{dim} \pi^{-1}(z)=m-s$ and $\pi_{Y}\left(\pi^{-1}(z)\right) \cap U \neq \emptyset$. For $a \in \pi_{Y}\left(\pi^{-1}(z)\right) \cap U$ let $H$ be an $(n-m+s)$-dimensional unirational subvariety of $Y$ intersecting $\pi_{Y}\left(\pi^{-1}(z)\right)$ isolatedly at $a$. Moreover, choose an $(m-s)$-dimensional affine subspace $L \subset \mathbb{C}^{m}$ such that $\{z\}$ is a component of $L \cap S$. We come to a contradiction as in Case 1, since $(H \times L) \cap W$ has components of dimension zero.

CASE 3: All fibers of $\pi: W \rightarrow \pi(W)$ have dimension $n$. Take $a \in Y$ such that $\operatorname{dim}\left(\left.\pi_{Y}\right|_{W}\right)^{-1}(a)=m-n$. Since $W$ is isomorphic to $\mathbb{C}^{m}$ we can find a variety $C \subset W$ which is isomorphic to $\mathbb{C}^{n}$ and $\left(\left.\pi_{Y}\right|_{W}\right)^{-1}(a) \cap C$ has dimension zero. Thus the projection $\pi_{Y}: C \rightarrow Y$ is dominant. This contradicts Lemma 1 because $Y$ dominates $X$.

The proof is complete.

COROLlaRY 2. Two stably equivalent hypersurfaces in $\mathbb{C}^{n}$ are equivalent if one of them is non-uniruled.

Corollary 3. Let $X \subset \mathbb{C}^{n}$ be a hypersurface which has only nonuniruled components. If $Y \subset \mathbb{C}^{n}$ is a hypersurface such that for some $m \in \mathbb{N}$ the cylinders $\left(\mathbb{C}^{n} \backslash X\right) \times \mathbb{C}^{m}$ and $\left(\mathbb{C}^{n} \backslash Y\right) \times \mathbb{C}^{m}$ are isomorphic then $X, Y$ are equivalent.

Proof. By Theorem 3 we have an induced isomorphism $f: \mathbb{C}^{n} \backslash X \rightarrow$ $\mathbb{C}^{n} \backslash Y$. By Lemma 1 the varieties $X$ and $Y$ have the same number of nonuniruled components. Using Hironaka's Theorem we can extend $f$ to a regular mapping $\tilde{f}: \mathbb{C}^{n} \backslash Z \rightarrow \mathbb{C}^{n}$, where $Z \subset \mathbb{C}^{n}$ has codimension at least 2 (compare the proof of Lemma 2). Once again we can extend $\tilde{f}$ to a regular mapping $\tilde{f}: \mathbb{C}^{n} \rightarrow \mathbb{C}^{n}$. Obviously $\tilde{f}$ does not have exceptional divisors so by the Zariski Main Theorem (see [8,9]), $\tilde{f}: \mathbb{C}^{n} \rightarrow \widetilde{f}\left(\mathbb{C}^{n}\right)$ is an isomorphism. Moreover $\widetilde{f}\left(\mathbb{C}^{n}\right)$ is Zariski-open in $\mathbb{C}^{n}$ and $\operatorname{dim}\left(\mathbb{C}^{n} \backslash \widetilde{f}\left(\mathbb{C}^{n}\right)\right) \leq n-2$ (because on $\widetilde{f}\left(\mathbb{C}^{n}\right)$ each invertible regular function is constant). Hence $\widetilde{f}^{-1}$ is regular on $\mathbb{C}^{n}$. Obviously $\widetilde{f}(X)=Y$.

Acknowledgements. I would like to express my gratitude to Professor Kamil Rusek for introducing me to the subject and helpful discussions. I also thank Professor Zbigniew Jelonek for useful comments. 


\section{References}

[1] A. van den Essen and V. Sphilrain, Some combinatorial questions about polynomial mappings, J. Pure Appl. Algebra 119 (1997), 47-52.

[2] H. Hironaka, Resolution of singularities of an algebraic variety over a field of characteristic zero, Ann. of Math. 79 (1964), 109-326.

[3] S. Iitaka and T. Fujita, Cancellation theorem for algebraic varieties, J. Fac. Sci. Univ. Tokyo 24 (1977), 123-127.

[4] Z. Jelonek, Irreducible identity sets for polynomial automorphisms, Math. Z. 212 (1993), 601-617.

[5] -, Test polynomials, J. Pure Appl. Algebra 147 (2000), 125-132.

[6] - Testing sets for properness of polynomial mappings, Math. Ann. 315 (1999), $1-35$.

[7] L. Makar-Limanov, P. van Rossum, V. Shpilrain and J. T. Yu, The stable equivalence and cancellation problems, Comment. Math. Helv. 79 (2004), 341-349.

[8] D. Mumford, Algebraic Geometry I, Springer, Berlin, 1976.

[9] I. R. Shafarevich, Basic Algebraic Geometry, Springer, Berlin, 1974.

[10] V. Shpilrain and J. T. Yu, Affine varieties with equivalent cylinders, J. Algebra 251 (2002), 295-307.

Institute of Mathematics

Jagiellonian University

Reymonta 4

30-059 Kraków, Poland

E-mail: Robert.Drylo@im.uj.edu.pl

Reçu par la Rédaction le 30.11.2004

Révisé le 21.6.2005 